arthritis domain both at month $6 \quad(\mathrm{p}<0.001)$ and 12 $(p<0.001)$. From baseline to month 6 , the mean tender joints count decreased from 5.7 to $2.7(\mathrm{p}=0.010)$, and the swollen joints count from 3.6 to $0.7(p<0.001)$; the decreases were sustained through month 12 ( $\mathrm{p}=0.001$ for both counts). No impact of smoking habits on treatment outcomes in relation to articular SLE was observed.

Conclusion In line with previous reports, belimumab treatment was effective in limiting mucocutaneous and articular symptoms in patients with SLE. A history of past or current smoking was found to reduce the efficacy of belimumab in mucocutaneous manifestations. Further survey on the impact of smoking on the efficacy of belimumab at a mechanistic level is merited.

\section{PS7:133 EXPOSURE-RESPONSE MODELLING AND EXPOSURE- SAFETY MODELLING ANALYSES IN TWO PHASE II STUDIES OF ATACICEPT IN SLE}

${ }^{1} \mathrm{O}$ Papasouliotis, ${ }^{1} \mathrm{O}$ Yalkinoglu, ${ }^{2} \mathrm{C}$ Vazquez Mateo, ${ }^{2} \mathrm{~S}$ Wax, ${ }^{2} \mathrm{~A}$ Kao, ${ }^{2} \mathrm{P}$ Chang, ${ }^{2} \mathrm{P}$ Fleuranceau-Morel, ${ }^{2} \mathrm{~L}$ Mahnke. ${ }^{1}$ Merck KGaA, Darmstadt, Germany; ${ }^{2} E M D$ Serono Research and Development Institute, Inc., Billerica, USA

\subsection{6/lupus-2018-abstract. 176}

Purpose Atacicept targets the B-cell stimulating factors BLyS and APRIL, and has been shown to reduce SLE disease activity.

Methods APRIL-SLE (NCT00624338) and ADDRESS II (NCT01972568) were phase II, multicenter studies in patients (pts) with autoantibody-positive SLE randomised (1:1:1) to weekly SC injections of atacicept (75 or $150 \mathrm{mg}$ ) or placebo (PBO). In APRIL-SLE, pts had BILAG A/B flare at Screening that was reduced to BILAG $\mathrm{C} / \mathrm{D}$ before randomization using corticosteroids; the primary endpoint was BILAG A/B flare over 52 weeks. In ADDRESS II, pts had SLEDAI- $2 \mathrm{~K} \geq 6$ at Screening; the primary endpoint was SRI-4 response at Week 24. SLE responder index (SRI)-6 response was analysed posthoc in high disease activity (HDA; SLEDAI-2K $\geq 10$ ) pts. Population pharmacokinetic (PK) model-derived exposure vs the probability of response (BILAG A/B flare, SRI-4, SRI-6), exploratory analysis of exposure vs safety, and population model simulations of serum IgG were analysed.

Results Exposure-response modelling suggests a relationship between atacicept exposure and SLE clinical response [figure 1], including serum IgG changes from baseline. The optimal atacicept exposure was AUCtau,ss $\geq \sim 1 \mathrm{mg} . \mathrm{hr} / \mathrm{mL}$, which is more achievable with weekly SC doses of atacicept $150 \mathrm{mg}$ than $75 \mathrm{mg}$ across a range of body weights. Body weightbased dosing is unlikely to offer any value over a fixed $150 \mathrm{mg}$ dose, based on comparable predicted clinical response. In HDA pts, greater reductions in serum $\operatorname{IgG}$ from baseline corresponded to a higher probability of SRI-6 response. Greater IgG reductions from baseline were associated with higher atacicept exposure; however, even at the highest exposure range, mean IgG reductions did not exceed $\sim 40 \%$. There was no association between serious/severe infections and exposure by PK quartile.

Conclusions Exposure-response modelling indicated robust relationships between atacicept exposure and clinical response or IgG levels, supporting the proposed mechanism of action for atacicept. Atacicept $150 \mathrm{mg}$ weekly SC is likely to provide an effective level of exposure with an acceptable safety profile. There was no evidence of an increased risk of severe or serious infections at higher exposures. Based on these results, the $150 \mathrm{mg}$ dose merits further evaluation.

\section{PS7:134 RITUXIMAB-MEDIATED LATE-ONSET NEUTROPENIA IN SYSTEMIC LUPUS ERYTHEMATOSUS - DISTINCT ROLES OF BAFF AND APRIL}

I Parodis, F Söder, F Faustini, Z Kasza, F Wermeling, I Gunnarsson. Karolinska Institutet, Stockholm, Sweden

\subsection{6/lupus-2018-abstract.177}

Background Rituximab-mediated late-onset neutropenia (LON) has been described in various diseases. We investigated its prevalence and contributing factors, including B cell related cytokines and growth factors of the myeloid lineage, in patients with systemic lupus erythematosus (SLE).
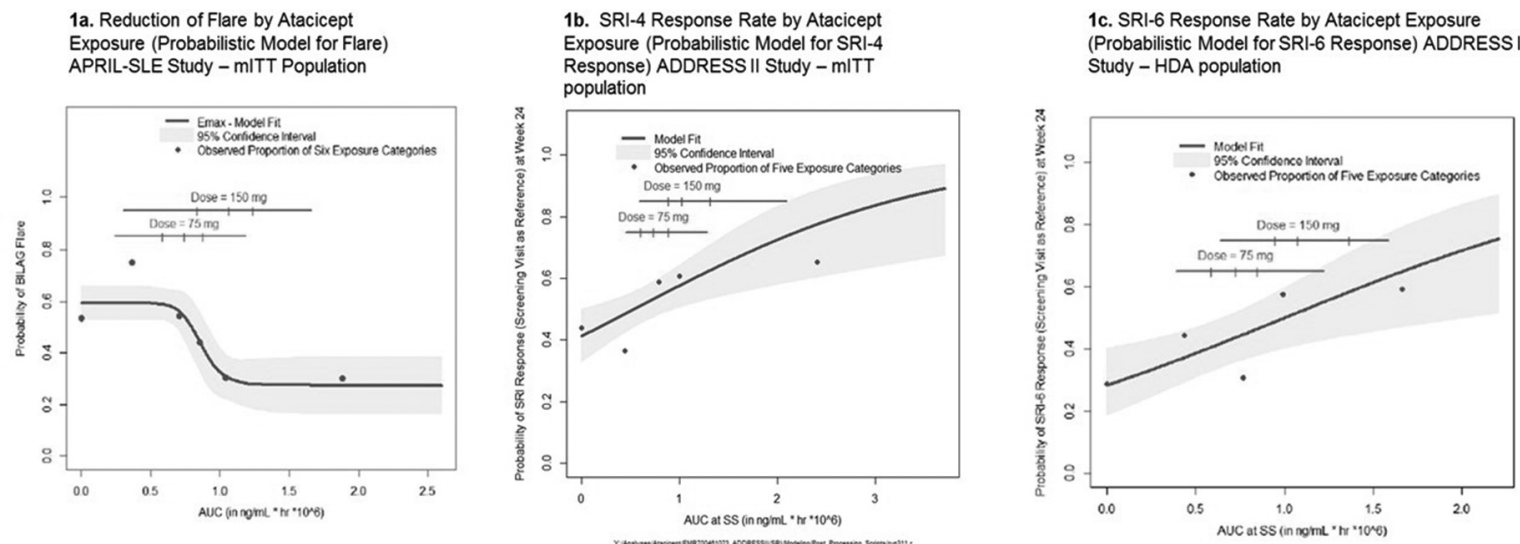

Exposure categories correspond to placebo (AUCtau $=0$ ) and quintiles (APRIL-SLE - 1a) or quartiles (ADDRESS $\|-1 \mathrm{~b}$ and $\mathrm{c}$ ) of the AUCtau distribution for subjects on atacicept Observed proportions (blue points) are plotted at the mid-point of the corresponding AUCtau exposure group. Solid blue lines are predicted mean profiles with shaded areas for $95 \%$ Observed proportions confidence intervals.
Horizontal lines correspond to the $95 \%$ of the distribution of AUCtau by dose. The 3 ticks are the 1st, 2 nd (median) and $3 r d$ quartiles.

AUCtau, area under the concentration curve over 1 dosing interval, ie, 1 week; BILAG, British Isles Lupus Assessment Group; BLyS, B lymphocyte stimulator; Emax, maximum response achievable; HDA, high disease activity (SLEDAl-2K $\geq 10$ at Screening); mITT, modified Intention-to-Treat (all randomized subjects who received at least 1 dose of investigational medicinal product); SLEDAI, Systemic Lupus Erythematosus Disease Activity Index; SRI-6, Systemic Lupus Erythematosus Responder Index 6 
Methods Rituximab-treated patients from the Karolinska University Hospital $(n=107)$ were enrolled. LON was defined as an absolute neutrophil count $<1,500$ cells/ $\mu \mathrm{L}$, occurring four weeks to two years following treatment, or later in cases of sustained B cell depletion, provided that other apparent causes were excluded. B cell activating factor (BAFF), a proliferationinducing ligand (APRIL), IL-6, granulocyte-macrophage colonystimulating factor (GM-CSF), and granulocyte colony-stimulating factor (G-CSF) were measured using ELISA prior to and post-treatment.

Results Thirty-two patients (29.9\%) developed LON after a median time of 201.5 days (IQR: 66.8-322.0). Thirteen patients were admitted to the hospital; ten developed fever, and three developed critical conditions. BAFF levels increased from baseline (median: $0.62 \mathrm{ng} / \mathrm{mL}$; IQR: 0.42-1.07) through the post-treatment measurement, both in patients who developed LON (median: $1.73 \mathrm{ng} / \mathrm{mL}$; IQR: 1.03-2.56; $\mathrm{p}=0.005$ ) and patients who did not (median: 1.03 ; IQR: $0.65-1.55$; $\mathrm{p}=0.001$ ), with significantly higher BAFF levels in the LON group $(p=0.021)$. APRIL levels were higher in the LON group both at baseline (median: 1.54 versus $1.15 \mathrm{ng} / \mathrm{mL} ; \mathrm{p}=0.027$ ) and post-treatment (median: 2.39 versus $1.11 \mathrm{ng} / \mathrm{mL} ; \mathrm{p}=0.011)$. IL-6 and GM-CSF levels decreased in the non-LON group $(\mathrm{p}<0.001)$. Cumulative rituximab and cyclophosphamide doses were found to be associated with the development of agranulocytosis $(p=0.022$ and $p=0.021$, respectively).

Conclusion Post-rituximab LON is a common complication in SLE. Although the phenomenon was self-limiting in most cases, a few patients developed life-threatening conditions; this highlights the importance of regular surveillance for neutrophil counts, fever and infections. Distinct roles of BAFF and APRIL are implicated; BAFF might contribute to granulopoiesis disruptions, whereas APRIL might have a value in distinguishing predisposed patients.

\section{PS7:135 AMG 592 IS AN INVESTIGATIONAL IL-2 MUTEIN THAT INDUCES HIGHLY SELECTIVE EXPANSION OF REGULATORY T CELLS}

${ }^{1} \mathrm{~N}$ Tchao, ${ }^{2} \mathrm{KS}$ Gorski, ${ }^{3} \mathrm{~T}$ Yuraszeck, ${ }^{4} \mathrm{SJ}$ Sohn, ${ }^{5} \mathrm{~K}$ Ishida, ${ }^{6} \mathrm{H}$ Wong, ${ }^{7} \mathrm{~K}$ Park. ${ }^{7}$ Amgen Inc., Department of Early Development, South San Francisco, USA; ${ }^{2}$ Amgen Inc., Department of Clinical Biomarkers, South San Francisco, USA; ${ }^{3}$ Amgen Inc., Department of Pharmacokinetics and Drug Metabolism, Thousand Oaks, USA; ${ }^{4}$ Amgen Inc., Department of Inflammation Research, South San Francisco, USA; ${ }^{5}$ Amgen Inc., Department of Comparative Biology and Safety Sciences, Thousand Oaks, USA; ${ }^{6}$ Amgen Inc., Department of Clinical Pharmacology, South San Francisco, USA; ${ }^{7}$ Amgen Inc., Department of Global Safety, Cambridge, USA

\subsection{6/lupus-2018-abstract. 178}

Background AMG 592 is an investigational IL-2 mutein designed for greater regulatory T-cell (Treg) selectivity and longer half-life than recombinant IL-2 (aldesleukin). We investigated the tolerability of AMG 592 and its effects on expansion of Tregs, conventional effector T-cells (Tcon), and natural killer (NK) cells.

Methods AMG 592 activity, in comparison with aldesleukin, was assessed by in vitro phosphorylated STAT5 (pSTAT5), Treg/Tcon/NK cell expansion, and cytokine production in primary human peripheral blood mononuclear cells (hPBMC). Effects on body temperature (Temp), C-reactive protein (CRP), and peripheral Treg/Tcon/NK cell numbers were evaluated in cynomolgus monkeys (CM). In an FIH study, healthy volunteers received single ascending SC AMG 592 doses $(n=6 /$ dose;
8 cohorts) or placebo $(n=2 /$ dose $)$ for 28 days. Adverse events (AEs), pharmacokinetics (PK), pharmacodynamics, and cytokines were evaluated.

Results In hPBMC cultures, AMG 592 caused more selective Treg response (pSTAT5, proliferation) and lower proinflammatory cytokine levels than aldesleukin. Dose-dependent expansion of FoxP3 + Tregs was associated with increased Temp and CRP in aldesleukin-treated but not in AMG 592-treated CM. In the FIH study, AMG 592 was well tolerated, with no serious AEs. The most common AE across cohorts was grade 1 painless erythema at/near the injection site which resolved without treatment. Preliminary PK results indicate dose-related increases in AMG 592 serum exposure. AMG 592 caused robust, dose-dependent Treg expansion relative to Tcon in all treated individuals. Expanded Tregs had increased CD25 and FoxP3, and were enriched for recent thymic emigrants. At the highest dose, increase in Treg: Tcon ratio peaked at day 8 ( $\sim 4$ fold vs baseline) and remained elevated up to day 29. AMG 592-mediated Treg expansion was highly selective, with no directional change in NK cell numbers and minimal increase in Tcon; there were no increases in serum proinflammatory cytokines IL-6, TNF $\alpha$, or IFN- $\gamma$ above the limits of detection.

Conclusion AMG 592 caused dose-dependent, selective Treg expansion in healthy volunteers. Lack of proinflammatory cytokines and reduced inflammation markers suggest a wider therapeutic margin, and sustained Treg elevation implies less frequent dosing, compared with aldesleukin. Further investigation of AMG 592-induced Treg-mediated restoration of immune homeostasis in inflammatory and autoimmune diseases is warranted.

\section{PS7:136 APOPTOTIC EFFECT OF BLYS ON ENDOTHELIAL CELLS AND ENDOTHELIAL PROGENITOR CELLS IS MEDIATED BY BLYS RECEPTORS AND IS REVERTED BY BELIMUMAB}

FR Spinelli, C Barbati, F Ceccarelli, T Colasanti, L Massaro, F Morello, C Garufi, C Alessandri, G Valesini, F Conti. Sapienza Università di Roma - Dipartimento di Medicina Interna e Specialità Mediche, Reumatologia, Rome, Italy

\subsection{6/lupus-2018-abstract.179}

Circulating endothelial progenitor cells (EPCs) are markers of endothelial function; their reduction and functional impairment in patients with Systemic Lupus Erythematosus (SLE), partially account for endothelial dysfunction. In murine models of atherosclerosis, treatment with a B Lymphocyte Stimulator (BLyS) reduced atherosclerotic plaque size and progression.

In a case study on SLE 20 women, our group confirmed a decrease in the number of EPCs, with a significant increase after treatment with Belimumab (BLM).

The aims of this study were: to evaluate the ex vivo and in vitro effects of BLyS stimulation and inhibition on the EPC colonies and on endothelial cells; to investigate BLyS receptor expression of on EPCs and endothelial cells.

EPCs were isolated from peripheral blood mononuclear cells and defined as CD34+/VEGF-R2 + double positive cells. To evaluate the ability to form colonies, the EPCs of 2 SLE patients and 2 healthy controls were cultured on fibronectincoated dishes and incubated with BlyS or Blys and BLM and counted after 7 days.

Apoptosis of EPCs and endothelial cell line (EA.hy926) was evaluated after 6, 12 and 24 hours incubation with BLyS and after 6 hour with BLyS and BLM. EPC and EA.hy926 were 